\title{
The B' Protein Phosphatase 2A Regulatory Subunit well- rounded Regulates Synaptic Growth and Cytoskeletal Stability at the Drosophila Neuromuscular Junction
}

\author{
Natasha M. Viquez, Caroline R. Li, Yogesh P. Wairkar, and Aaron DiAntonio \\ Department of Molecular Biology and Pharmacology, Washington University School of Medicine, St. Louis, Missouri 63110
}

\begin{abstract}
Synaptic growth is essential for the development and plasticity of neural circuits. To identify molecular mechanisms regulating synaptic growth, we performed a gain-of-function screen for synapse morphology mutants at the Drosophila neuromuscular junction (NMJ). We isolated a $\mathrm{B}^{\prime}$ regulatory subunit of protein phosphatase $2 \mathrm{~A}(\mathrm{PP} 2 \mathrm{~A})$ that we have named well-rounded $(\mathrm{wrd})$. Neuronal overexpression of wrd leads to overgrowth of the synaptic terminal. Endogenous Wrd protein is present in the larval nervous system and muscle and is enriched at central and neuromuscular synapses. wrd is required for normal synaptic development; in its absence, there are fewer synaptic boutons and there is a decrease in synaptic strength. wrd functions presynaptically to promote normal synaptic growth and postsynaptically to maintain normal levels of evoked transmitter release. In the absence of $\mathrm{wrd}$, the presynaptic cytoskeleton is abnormal, with an increased proportion of unbundled microtubules. Reducing PP2A enzymatic activity also leads to an increase in unbundled microtubules, an effect enhanced by reducing $w r d$ levels. Hence, $w r d$ promotes the function of PP2A and is required for normal cytoskeletal organization, synaptic growth, and synaptic function at the Drosophila NMJ.
\end{abstract}

Key words: PP2A; B' subunit; synaptogenesis; cytoskeleton; Drosophila; neuromuscular junction

\section{Introduction}

The regulation of neuronal growth is vital for the development and refinement of neural circuits. The Drosophila larval neuromuscular junction (NMJ) is a useful system for identifying genes controlling neuronal growth, because mutations that alter the stereotyped morphology of the larval NMJ are readily identified. To search for novel regulators of synaptic growth, we performed a gain-of-function screen for genes whose neuronal overexpression affects growth at the Drosophila NMJ. We recovered a regulatory $\mathrm{B}^{\prime}$ subunit of protein phosphatase $2 \mathrm{~A}(\mathrm{PP} 2 \mathrm{~A})$ and have characterized its role in synaptic development.

Protein phosphatase $2 \mathrm{~A}$ is a ubiquitous serine-threonine phosphatase required for many fundamental cellular processes, including mitosis, apoptosis, and the regulation of cell morphology (Janssens and Goris, 2001). The PP2A holoenzyme comprises three subunits: a catalytic subunit and a structural subunit, which comprise the core enzyme, and a regulatory subunit. Four structurally distinct families of regulatory subunits, $\mathrm{B}, \mathrm{B}^{\prime}, \mathrm{B}^{\prime \prime}$, and $\mathrm{B}^{\prime \prime \prime}$, can associate with the core PP2A enzyme. The catalytic subunits of PP2A are highly conserved from yeast to human, whereas regulatory subunits are more diverse and likely govern the subcellu-

Received Dec. 20, 2005; revised July 13, 2006; accepted Aug. 1, 2006.

This work was supported by National Institute of Neurological Disorders and Stroke Grant NS043171, the Keck Distinguished Scholars Program, and the McKnight Scholars Award (A.D.). We thank Scott Portman and Angela Wilkinson for excellent technical assistance and Cathy Collins and Sylvia Johnson for comments on this manuscript.

Correspondence should be addressed to Dr. Aaron DiAntonio, Department of Molecular Biology and Pharmacology, Campus Box 8103, 660 South Euclid, Washington University School of Medicine, St. Louis, M0 63110. E-mail: diantonio@wustl.edu.

DOI:10.1523/JNEUROSCI.1740-06.2006

Copyright $\odot 2006$ Society for Neuroscience $\quad$ 0270-6474/06/269293-11\$15.00/0 lar localization and substrate specificity of PP2A (Janssens and Goris, 2001).

Although PP2A is present in all tissues, it is highly enriched in the nervous system, in which it localizes to central synapses (Betz and Henkel, 1994; Barnes et al., 1995) and associates with neuronal microtubules (Sontag et al., 1995; Price et al., 1999). Regulatory subunits are differentially expressed in the vertebrate brain, suggesting that they control specific aspects of PP2A function in the nervous system (Strack et al., 1998). Several studies of the role of PP2A in the brain have implicated PP2A in the regulation of long-term potentiation (Woo and Nguyen, 2002; Jouvenceau et al., 2003; Belmeguenai and Hansel, 2005). PP2A is also postulated to contribute to the pathogenesis of Alzheimer's disease, because reduced PP2A activity is linked to increased levels of hyperphosphorylated tau (Sontag et al., 1996, 1999; Vogelsberg-Ragaglia et al., 2001). Despite the prominent expression of PP2A in the developing nervous system and its localization at synapses, little is known regarding the role of PP2A and its regulatory subunits in synaptic development.

Here, we describe gain- and loss-of-function synaptic phenotypes for a Drosophila B' regulatory subunit, which we have named well-rounded ( $w r d)$. Neuronal overexpression of $w r d$ promotes synaptic growth. The endogenous Wrd protein is widely expressed and is enriched at both central and neuromuscular synapses. Loss-of-function mutants in $w r d$ have reduced bouton number and decreased synaptic strength. wrd is required presynaptically for normal synaptic growth and postsynaptically for normal levels of evoked transmitter release, implying a role in the retrograde control of synaptic function. wrd mutants additionally 
demonstrate an increased proportion of unbundled synaptic microtubules. PP2A activity is also required for proper synaptic microtubular organization, a function promoted by $w$ rd. Hence, wrd is required for normal synaptic growth and morphology and likely mediates these functions by positively regulating PP2A activity.

\section{Materials and Methods}

Genetics. Flies were raised on standard food at $25^{\circ} \mathrm{C}$. Wild-type (WT) flies were Canton $\mathrm{S}$ outcrossed to either the neuron-specific elav (embryonic lethal, abnormal vision, Drosophila) Gal4 driver (Yao and White, 1994) or the muscle-specific G7 Gal4 driver (Zhang et al., 2001). The P-element insert lines were generated using the gene search P-element designed with upstream activating sequence (UAS) promoters flanking both ends (Toba et al., 1999). A Gene Search P-element on the X chromosome was mobilized to generate hops onto the second and third chromosome, which included insertions into the $\mathrm{B}^{\prime}$ gene. The transgenic UAS wrd line was made by cloning a cDNA of the RD splice form of wrd (see Fig. 1a) into the pUAST vector (Brand and Perrimon, 1993). The wrd mutants $w r d^{104}$ and $\mathrm{Df}(3 \mathrm{R}) 189$ were generated by imprecise P-element excision. The futschK68 mutants were obtained from Graeme Davis (University of California, San Francisco, CA) (Roos et al., 2000). The UAS Mts (microtubule star), UAS dominant-negative (dn) Mts, UAS Wdb (Widerborst), and UAS dnWdb lines were obtained from Suzanne Eaton (Max Planck Institute, Dresden, Germany) (Hannus et al., 2002). The deficiency line Df(3R)DG4 was obtained from Bloomington Stock Center (Bloomington, IN).

Antibody generation. A polyclonal rabbit antibody to Wrd was raised against a 20 residue peptide sequence, DQYSENDSDAYDQSEQRARQ, from exon 11, which is predicted to be present in all splice forms of wrd (Zymed, San Francisco, CA). The secondary bleed was affinity purified against the peptide, and the KSCN elution was used for all tissue staining and Western blots.

Immunohistochemistry. Wandering third-instar larvae were dissected in ice-cold PBS and fixed in either 4\% formaldehyde or Bouin's fixative for $7 \mathrm{~min}$. Larvae were then washed with PBS containing $0.1 \%$ Triton $\mathrm{X}-100$ (PBT) and blocked in 5\% NGS in PBT for 30 min. Blocking was followed by incubation in primary antibodies in 5\% NGS in PBT ranging from $1 \mathrm{~h}$ to overnight, three washes for a total of $30 \mathrm{~min}$ in PBT, incubation in secondary antibodies in 5\% NGS in PBT for $1 \mathrm{~h}$, three final washes in PBT, and equilibration in 70\% glycerol in PBS. A slightly modified protocol was performed for the $\alpha$-Wrd antibody, in which PBS rather than PBT was used for incubation in the secondary antibodies as well as the final washes. The following primary antibodies were used: rabbit $\alpha$-Wrd at 1:1000, rabbit $\alpha$-Drosophila vesicular glutamate transporter (DVGLUT) at 1:10000 (Daniels et al., 2004), rabbit $\alpha$-glutamate receptor III at 1:2000 (Marrus et al., 2004), mouse $\alpha$-Bruchpilot at 1:100 (obtained from Eric Buchner, Theodor Boveri Institute, Wurzburg, Germany) (Wagh et al., 2006), mouse $\alpha$-acetylated tubulin at 1:1000 (Sigma, St. Louis, MO), and cyanine 5 (Cy5)-conjugated goat $\alpha$-HRP at 1:1000 (Jackson ImmunoResearch, West Grove, PA). The antibodies mouse $\alpha$-Discs large (Dlg) [monoclonal antibody (mAb) 4f3], developed by Corey S. Goodman (Renovis, San Francisco, CA), and mouse $\alpha$-Futsch at 1:100 (mAb 22C1), developed by Seymour Benzer (California Institute of Technology, Pasadena, CA), were obtained from the Developmental Studies Hybridoma Bank, developed under the auspices of the National Institute of Child Health and Human Development and maintained by the Department of Biological Sciences of the University of Iowa (Iowa City, IA). Secondary antibodies Cy3-conjugated goat $\alpha$-mouse and Alexa 488 goat $\alpha$-rabbit (Jackson ImmunoResearch) were used at dilutions of $1: 1000$.

Western blots. Second-instar larvae were homogenized in ice-cold homogenization buffer (67 mm Tris- $\mathrm{HCl}, \mathrm{pH} 8.0,67 \mathrm{~mm} \mathrm{NaCl}, 2 \mathrm{~m}$ urea, 1 mM EDTA, and $1.3 \%$ SDS), and samples were run on $8 \%$ SDS-PAGE gels according to standard procedures. Rabbit $\alpha$-Wrd was used at 1:1000. HRP-conjugated goat $\alpha$-rabbit secondary antibody (Jackson ImmunoResearch) was used at 1:10,000.

Imaging and analysis. Samples were imaged using a Nikon (Tokyo,
Japan) C1 confocal microscope. For detection of Wrd staining, wild-type and mutant samples were imaged at the same gain. For all other data, images were taken at the maximum level of brightness, which avoided saturation. Images were analyzed using MetaMorph software (Universal Imaging Corporation, West Chester, PA). The experimenter was blinded to genotypes during both imaging and analysis. Statistical analysis was performed using one-way ANOVA for comparison of samples within an experimental group. $n$ for each condition is described in the figures. All histograms and measurements are shown as mean \pm SEM.

Bouton analysis. Quantification of bouton number and size was performed as described previously (Wu et al., 2005) using confocal images analyzed with MetaMorph software (Universal Imaging Corporation). Wandering third-instar larvae were costained for antibody to HRP and DVGLUT. HRP staining labels the neuronal membrane, whereas DVGLUT stains synaptic vesicles and highlights the cytoplasm of synaptic boutons. Boutons were counted by identifying rounded profiles at the synaptic terminal having both HRP staining and strong DVGLUT staining. To quantify bouton size, DVGLUT staining was thresholded to a common value for all images in an experimental cohort. All boutons analyzed were from $1 \mathrm{~b}$ innervation on muscle 4 . Muscle segments A2-A4 were analyzed, and muscle size for all genotypes was similar. The MetaMorph area function was then used to measure the thresholded region of each bouton.

Microtubule unbundling assay. Measurements of synaptic cytoskeletal organization were based on confocal images of larvae costained with antibody to Futsch, a neuron-specific microtubule-associated protein (MAP), and the antibody to DVGLUT, which highlights the cytoplasm of the synaptic terminal. Futsch staining, which colocalizes with $\alpha$-tubulin at the NMJ (Roos et al., 2000), was used as a reporter for presynaptic microtubules at the NMJ. Because Futsch staining varies widely in intensity throughout the synaptic terminal, images were obtained at two intensities for each NMJ. A low-intensity image was taken to avoid saturating the strong Futsch signal within the core region of the synaptic terminal, whereas a high-intensity image allowed visualization of fainter Futsch staining at the terminal boutons. (For ease of presentation in Figures 6 and 7, all Futsch images in a given figure were taken at the same gain, which was set for a moderate level of Futsch intensity.) Images were analyzed using MetaMorph software, and the experimenter was blinded during both imaging and analysis. Only Futsch-positive areas that overlapped with DVGLUT staining were counted as synaptic microtubules. In MetaMorph, synaptic Futsch was thresholded to a common value for each experimental cohort. Thresholded areas were classified as one of two types of microtubule regions: unbundled microtubule regions, which included looped, splayed, or punctuate Futsch staining (Packard et al., 2002), or bundled microtubule regions. The area of the unbundled and bundled microtubule regions was measured with MetaMorph. An index of microtubule unbundling was then calculated by dividing the area of unbundled microtubule regions by the total area of synaptic microtubules at the NMJ [unbundled microtubules/(unbundled + bundled microtubules)].

Electrophysiology. Intracellular electrophysiological recordings were done as reported previously (Marrus and DiAntonio, 2004; Marrus et al., 2004). Briefly, wandering third-instar larvae were dissected in $0.5 \mathrm{~mm}$ $\mathrm{Ca}^{2+}$ Stewart saline (HL3) (Stewart et al., 1994). Both spontaneous miniature excitatory junction potentials (mEJPs) and evoked potentials (EJPs) were then recorded in $0.5 \mathrm{~mm}$ HL3. At least 60 consecutive miniature events were measured per cell using MiniAnalysis (Synaptosoft, Decatur, GA) and averaged to determine mean mEJP. Events with a slow rise time were rejected as artifacts from neighboring electrically coupled muscle cells. To record evoked EJPs, segmental nerves were cut and suctioned into a stimulating electrode, in which they received a brief ( 1 $\mathrm{ms}$ ) depolarizing pulse. Quantal content was estimated by dividing the mean EJP by the mean mEJP. Cells across all genotypes had similar mean input resistances and resting potentials. Quantal size was not significantly different among any of the genotypes compared with wild-type controls [ $\mathrm{wrd}^{104} / \mathrm{D} f(3 R) D G 4, p>0.3$; all other genotypes, $\left.p>0.9\right]$. Statistical analysis was performed using one-way ANOVA to compare samples in an experimental group. 


\section{Results}

\section{Neuronal overexpression of well-rounded promotes} synaptic growth

To identify molecules that can regulate synaptic development, we performed a gain-of-function screen for genes whose overexpression disrupts the stereotyped morphology of identified motoneurons at the Drosophila NMJ. We generated a collection of $>2500$ transposable P-element insertion lines in which the yeast UAS promoter was randomly inserted into the genome (Rorth et al., 1998; Toba et al., 1999). When crossed to flies expressing the yeast transcription factor Gal4, these lines express the gene adjacent to the P-element insertion exclusively in tissues that express Gal4. We designed our screen to enrich for genes that affect synapse development. First, we drove P-element insertion lines with elav Gal4 to limit expression to postmitotic neurons (Yao and White, 1994). Second, we identified lines whose neuronal expression enhanced a known synaptic morphology mutant to lethality. We demonstrated previously that neuronal overexpression of fat facets leads to synaptic overgrowth and that mutations in a second synapse morphology gene hiw (highwire) are lethal in combination with overexpressed fat facets (DiAntonio et al., 2001). We reasoned that other genes with synapse morphology phenotypes might also be lethal in combination with overexpressed fat facets. To identify such enhancers, we selected lines meeting two conditions: (1) the lines are lethal when coexpressed with fat facets in neurons but (2) the lines are viable on their own when driven in neurons. For each enhancer, we performed a secondary screen to identify lines that affect synapse morphology without a requirement for coexpressing fat facets. We assayed synapse morphology at third-instar NMJs using an antibody that recognizes neuronal membranes ( $\alpha$-HRP) and retained those lines whose neuronal overexpression disrupts synapse morphology. This collection includes four lines in which the $\mathrm{P}$-element insertion is positioned to overexpress a regulatory $\mathrm{B}^{\prime}$ subunit of PP2A, which we dubbed well-rounded ( $w r d$ ) based on its loss-of-function phenotype (see below).

The wrd gene, previously annotated as CG 7913, is one of two $\mathrm{B}^{\prime}$ regulatory subunits in the Drosophila genome. There are five wrd splice forms, labeled RA-RE, all of which share a common C terminus encoded by exons 7-10 that includes the B56 domain that is the defining feature of $\mathrm{B}^{\prime}$ subunits (Fig. 1a). Splice forms can be subdivided into two groups with very different $\mathrm{N}$ termini (Fig. 1a): (1) RC, RD, and RE splice forms, which can include exons 1-3 and (2) the RA and RB splice forms, which include exons 4-6. Multiple P-element insertions were identified that are predicted to overexpress both classes of transcripts (Fig. 1a).

Overexpression of $w r d$ in neurons leads to synaptic overgrowth (Fig. 1b). To quantify this phenotype, we analyzed synaptic area, bouton size, and bouton number at NMJs on muscle 4 in wandering third-instar larvae. We restricted our quantitative analysis to the synapse from a single, identified motoneuron, MN4-1b (Hoang and Chiba, 2001), although the phenotype is qualitatively similar at all type I neuromuscular junctions, which are the primary excitatory glutamatergic synapses at the NMJ. We defined the synaptic area based on the localization of a synaptic vesicle protein, DVGLUT, that is present at glutamatergic synapses (Daniels et al., 2004). Overexpression of $w$ rd leads to a $79 \%$ increase in the area of the synaptic terminal (WT, $100 \pm 0.19 \%$ of WT, $n=12$; elav/P [676], $179 \pm 0.34 \%$ of WT, $n=12 ; p<0.05)$. In Drosophila, the NMJ is organized into synaptic boutons. In addition to increasing synapse size, overexpression of $w \mathrm{rd} \mathrm{im-}$ pairs normal synaptic bouton development, leading to a $178 \%$ a

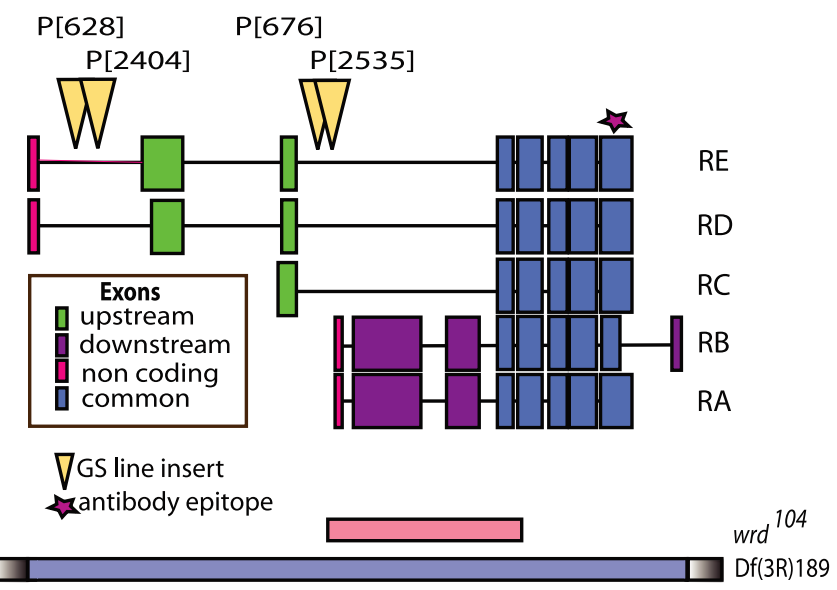

b

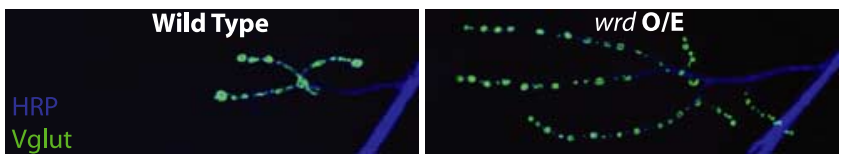

Figure 1. well-rounded promotes synaptic growth. $\boldsymbol{a}$, Gene structure of well-rounded (CG 7913). well-rounded has five splice forms. Exons are designated by boxes. Blue boxes indicate exons common to all five splice forms, pink boxes designate noncoding exons, green boxes designate a cluster of exons present in three of the wrd splice forms, and lavender boxes designate a second cluster of exons present in two of the wrd splice forms. Triangles indicate P-element insertions obtained in gain-of-function screen (GS). Imprecise excision of $P$ [2535] was used to generate the wrd $^{104}$ and Df(3R) 189 mutants, whose deletions are illustrated by bars at the bottom. rrd $^{104}$ is missing exons 4 and 5 as well as some exons common to all splice forms, whereas Df(3R)189 removes all of the wrd gene and five adjacent genes. Star marks the location of the antigen for the Well-rounded antibody. $\boldsymbol{b}$, Representative confocal images of muscle 4 synapses from wild-type third-instar larvae (elav Gal4/+) and wrd overexpressing (0/E) third instars, which express additional wrd via the P[676] insert driven pan-neuronally with elav Gal4 (elav Gal4/P[676]). Synapses are costained with antibodies to DVGLUT (green), which highlights the cytoplasm of the synaptic terminal, and HRP (blue), which highlights neuronal membranes.

increase in the number of boutons (WT, $14 \pm 0.1$; elav/P[676], $39 \pm 3.3, n=16 ; p<0.001)$ and a $63 \%$ decrease in their relative size (WT, $1.00 \pm 0.16, n=16$; elav/P[676], $0.37 \pm 0.01, n=16$; $p<0.001$ ) (Fig. $1 b$ ). Expression of $w r d$ from a second P-element, $\mathrm{P}[628]$, also leads to synaptic overgrowth, resulting in a $51 \%$ increase in bouton number $(p<0.01 ; n=16)$.

Although these P-element lines are convenient for identifying candidate genes that give gain-of-function phenotypes, they lead to overexpression of genes on both sides of the P-element insertion site. To test whether wrd is responsible for the gain-offunction phenotype, we generated a transgenic fly in which a wrd cDNA to the RD splice form is under the control of the UAS promoter. Neuronal overexpression of this wrd cDNA leads to synaptic overgrowth, with a $33 \%$ increase in bouton number (WT, mean of $18.1 \pm 0.9, n=36$; elav UAS $w r d$, mean of $24.4 \pm$ $1.2, n=36 ; p<0.05$ ) and a $20 \%$ decrease in bouton size (WT, mean of $33.7 \pm 0.9 \mu \mathrm{m}, n=36$; elav/UAS $w r d, 27.1 \pm 0.7 \mu \mathrm{m}$, $n=36$; $p<0.05$ ). Overexpression of $w r d$, therefore, can promote synaptic growth and regulate synaptic bouton morphology. Given that wrd is a regulatory subunit of PP2A, this finding suggests that PP2A and its substrates may regulate growth and development of the Drosophila NMJ. 


\section{Drosophila Wrd is enriched in the larval nervous system and} is present at the NMJ

The gain-of-function wrd phenotype could be attributable to either overexpression or misexpression of $w r d$ in the CNS. Because we are interested in molecules that normally regulate synaptic growth, we characterized the expression of endogenous $w$ rd to distinguish between these possibilities. RNA in situ hybridization demonstrates that $w r d$ is enriched in the embryonic nervous system, consistent with a role for $w r d$ in nervous system development (supplemental Fig. 1, available at www.jneurosci.org as supplemental material). To analyze the subcellular localization of the Wrd protein, we generated a polyclonal antibody to a peptide from exon 11 of the $w r d$ gene. The peptide antigen is common to all five $w r d$ splice forms and is absent from the other Drosophila $\mathrm{B}^{\prime}$ protein, Wdb, and all other predicted Drosophila proteins. The affinity-purified antiserum labels the nervous system, imaginal disks, salivary glands, and muscle in third-instar larvae. To confirm that the sera can recognize Wrd, we stained larvae overexpressing UAS $w r d$ in the nervous system. Staining intensity in brain was increased relative to wild type (Fig. $2 a$ ), as well as in axons and at the NMJ (Fig. 2b), confirming that the antibody can detect Wrd protein. In the preterminal axon, however, there is very little Wrd detected, even when Wrd is neuronally overexpressed (Fig. $2 b$, arrows), demonstrating that Wrd is enriched in synaptic boutons. In addition, the staining disappears in a wrd null mutant (described below).

Because the antisera is specific for Wrd, we used it to characterize Wrd localization in the nervous system. In the larval CNS, staining is observed in the brain lobes and ventral nerve cord and is enriched in the synaptic neuropil (Fig. 2a). Peripherally, staining is present in motoneuron axons and at the neuromuscular junction (Fig. 2b,c). We costained larvae with $\alpha$-Wrd and either $\alpha$-HRP, a presynaptic marker, or $\alpha$-Dlg, a primarily postsynaptic marker. The NMJ staining for Wrd overlaps well with $\alpha$-HRP at the NMJ, because the synaptic regions outlined by the two antibodies align (Fig. 2c). Although the majority of Wrd is presynaptic, lower levels of Wrd may be present in the postsynaptic compartment, because some Wrd staining overlaps with Dlg staining. Wrd staining is also present diffusely throughout the postsynaptic muscle. Because endogenous Wrd is in the presynaptic terminal of the NMJ, it is positioned to regulate PP2A activity locally at the synapse. The gain-of-function phenotype is therefore attributable to overexpression and not misexpression of $w r d$. The presence of Wrd at the NMJ and its ability to promote synaptic growth suggests that it may normally function to regulate synaptic development.

\section{Generation of loss-of-function wrd mutants}

We demonstrated that Wrd localizes to synapses and can promote synaptic growth. Although these expression and gain-offunction studies illustrate what $w r d$ can do, only loss-of-function analysis can demonstrate what $w r d$ actually does. To test whether wrd is required for normal synaptic growth, we generated lossof-function mutants. A P-element transposon, P[2535], located in the middle of the $w r d$ gene (Fig. $1 a$ ) was used to create imprecise excisions. Among the imprecise excisions, we generated a small deficiency, $\mathrm{Df}(3 \mathrm{R}) 189$, that deletes the entire wrd gene as well as five adjacent genes, CG 32920, CG 7215, CG 7217, CG 7212 , and CG 7208. Because the entire coding sequence for $w r d$ is absent, Df(3R)189 is a definitive null for wrd. Homozygous Df(3R)189 larvae are viable until the third-instar stage, after which they fail to pupate and then die. A mutant exclusively in the wrd gene, $w r d^{104}$, was also obtained in the P-element excision
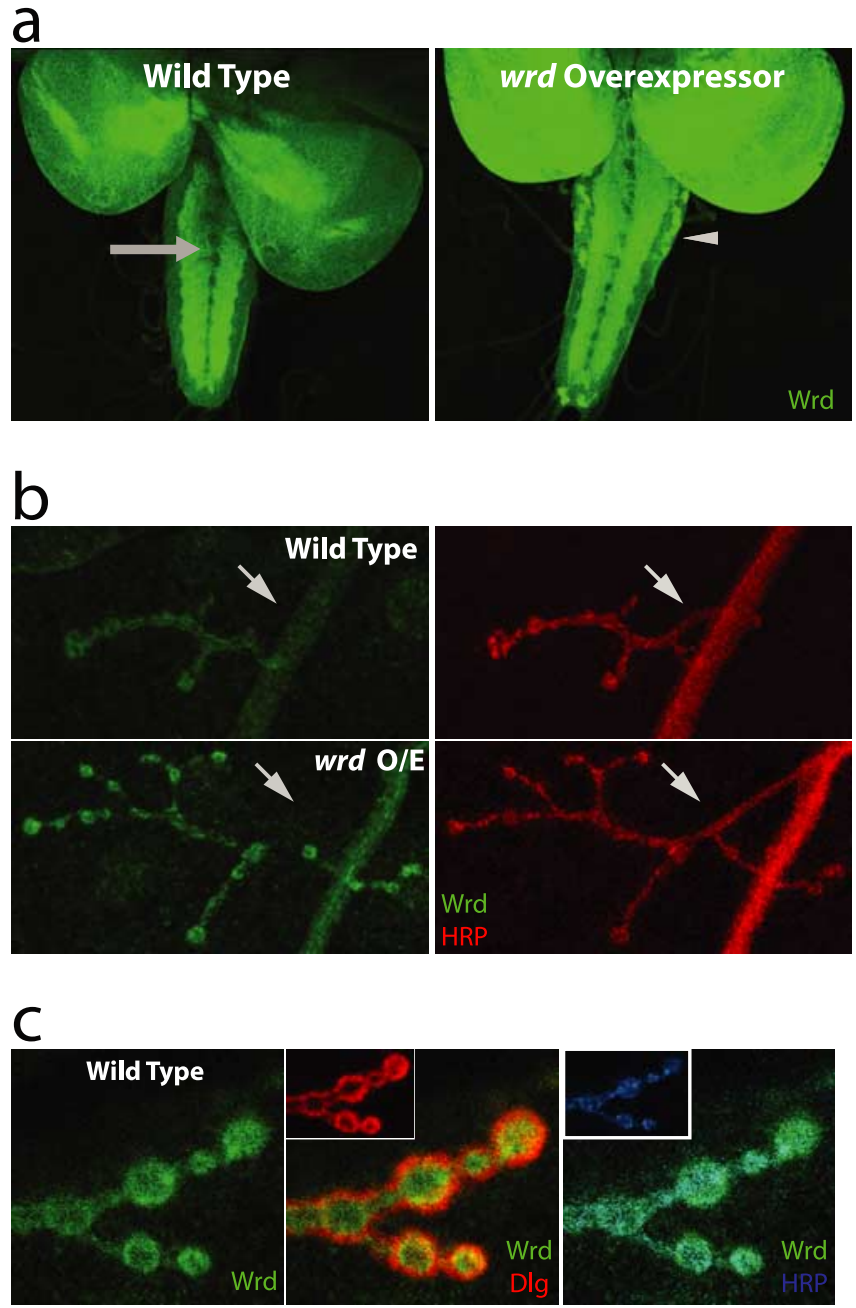

Figure 2. Well-rounded is present at synapses. $\boldsymbol{a}$, Confocal images of larval brains stained with polyclonal antibody to Wrd. Left displays a wild-type brain, and right displays a brain from an animal expressing a wrd cDNA driven pan-neuronally under elav Gal4. Wrd labeling is prominent in brain lobes and the synapse-rich region of neuropil (arrow, right). Overexpression leads to increased staining of brain lobes and cell bodies in the cortex of neuropil (arrowhead, left). $\boldsymbol{b}$, Confocal images of muscle 4 larval NMJs costained with antibody to Wrd (green) and the neuronal marker HRP (red). Top row displays an NMJ from a wild-type animal, and bottom row is from an animal with pan-neuronal expression of Wrd under elav Gal4. Overexpression (0/E) leads to increased Wrd staining at the NMJ. c, Costaining of Wrd at wild-type NMJ with presynaptic and postsynaptic markers. Image demonstrates a single confocal slice. Left, The NMJ is stained for Wrd alone (green). Middle, The same synapse is stained for Wrd and Dlg (red), a postsynaptic marker. Right, The synapse costained with Wrd and HRP (blue), a presynaptic marker. Synaptic Wrd staining colocalizes nearly completely with HRP, as demonstrated by the increased intensity throughout the NMJ in the overlay of Wrd and HRP. Overlay of Wrd and Dlg demonstrates partial localization, because a ring of Dlg staining falls outside the Wrd staining.

screen and is homozygous viable. In this mutant, the DNA encoding exon 4 through exon 7 is deleted. Because exon 7 is common to all five splice forms of $w r d, w r d^{104}$ is a candidate protein null.

To determine whether $w r d^{104}$ expresses Wrd protein, we performed immunoblot analysis from larvae of wild-type animals, the definitive null Df(3R)189, and $w r d^{104}$ homozygotes (hz). In wild-type larvae, several bands are observed as predicted by the presence of multiple $w r d$ splice forms (Fig. 3a). These bands are absent in the definitive null $\mathrm{Df}(3 \mathrm{R}) 189$, demonstrating that they are specific for the Wrd protein. These bands are also absent in $w_{r d} d^{104}$ homozygotes. Because the antibody is directed to an epitope downstream of the deletion, it could have detected any 
a
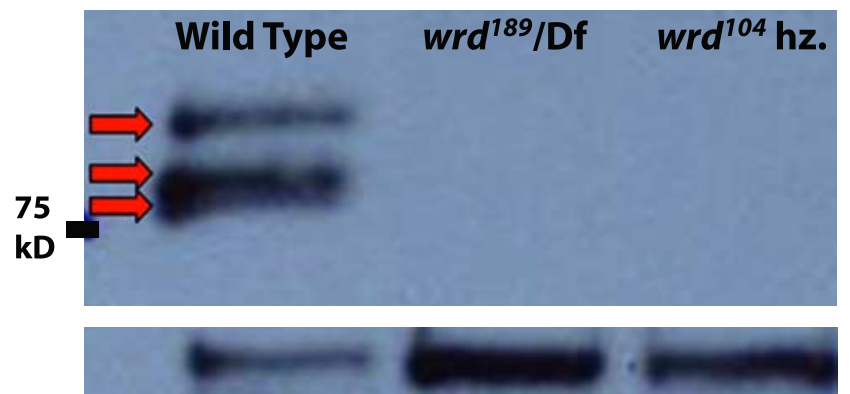

b

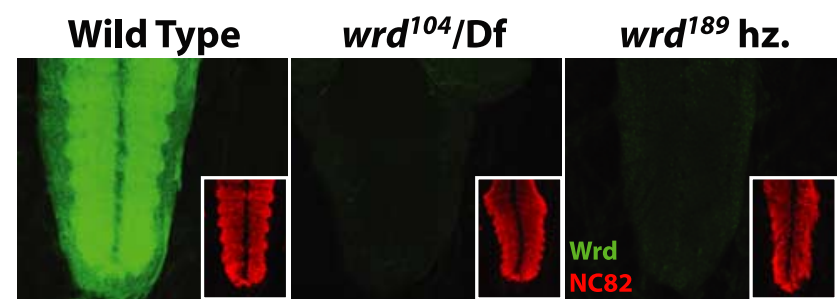

C
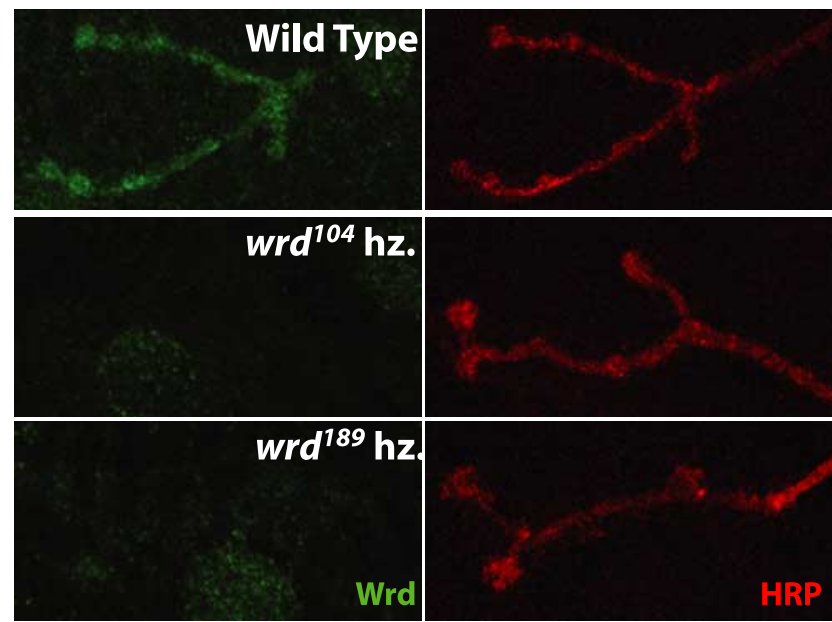

Figure 3. Generation of wrd loss-of-function mutants. $\boldsymbol{a}$, Western blot confirming absence of Wrd protein in excision mutants. Genotypes are as follows: wild type, elav Gal4/+; wrd $^{189} /$ Df(3R)DG4, Df(3R)189/Df(3R)DG4; and wrd ${ }^{104} \mathrm{hz}$, wrd ${ }^{104} /$ wrd $^{104}$. $\alpha$-Wrd reveals three bands (arrows) in wild-type animals, consistent with the presence of multiple wrd splice forms. These bands are absent in the lane for $\operatorname{wrd}^{189} / D f(3 R) D G 4$, a definitive null lacking the wrd gene, showing that the antibody specifically recognizes Wrd protein. The Wrd bands are also absent in the rrd $^{104}$ homozygote, which is therefore a protein null. Bottom half of blot shows a nonspecific background band, here shown as a loading control. $\boldsymbol{b}$, Representative neuropil staining for Wrd in wild-type animals, wrd ${ }^{104}$ mutants, and wrd ${ }^{189} \mathrm{hz}$ [Df(3R)189/Df(3R)189]. Animals were costained for Wrd and Bruchpilot, an active zone marker with strong neuropil staining. Bruchpilot staining is shown in insets. Absence of Wrd staining in wrd ${ }^{104}$ mutants and $w r d^{189} \mathrm{hz}$ demonstrates specificity of staining at synapses in neuropil. Remaining staining outside of neuropil represents background, because it persists in $w_{r d}{ }^{189} \mathrm{hz}$, a definitive null. c, Representative synaptic staining for Wrd at the NMJ on muscle 4 of wild-type animals, wrd ${ }^{104}$ mutants, and $w_{r d} d^{189} \mathrm{hz}$. The NMJ is stained for Wrd as well as HRP to delineate the synaptic terminal. Whereas Wrd staining is present at the NMJ in wild-type animals, it is essentially absent in the wrd $^{104}$ mutant and the $w^{18 d^{189}} \mathrm{hz}$, demonstrating specificity of Wrd staining at NMJ.

altered protein that included the $\mathrm{C}$ terminus. Therefore, only small, N-terminal protein fragments would not be detected by the antibody, and such fragments would encode none of the conserved B56 domain. Because the $w r d^{104}$ homozygotes live through adulthood, these results demonstrate that the $w r d$ gene is not required for viability. Tissue staining in $\mathrm{wrd}^{104}$ homozygotes confirms the loss of Wrd protein. For example, the synaptic staining in the neuropil is not detected in the $w r d^{104}$ mutant (Fig. 3b). Synaptic staining is also lost at the NMJ in the rrd $^{104}$ mutant (Fig. $3 c$ ). Although most tissue staining is lost in the wrd mutants, some does remain. Staining in the definitive null $\operatorname{Df}(3 R) 189$ is indistinguishable from $w r d^{104}$ (Fig. $3 b, c$ ), indicating that residual staining is attributable to cross-reactivity of the antibody with another antigen and not mutant Wrd protein. The staining that does disappear can be attributed to Wrd and confirms the enrichment of endogenous Wrd in the nervous system.

\section{wrd mutants alter synaptic morphology}

The generation of $w r d$ loss-of-function mutants allowed us to ask whether wrd is necessary for normal synaptic growth. Because overexpression of $w r d$ increases bouton number and decreases bouton size, we asked whether the wrd mutants affect bouton number and bouton size. We analyzed type $1 \mathrm{~b}$ boutons on muscle 4 in two different wrd mutants: $w_{r d} d^{104}$ homozygotes and the wrd $^{104}$ mutation in combination with an independently derived deficiency line, $D f(3 R) D G 4$, that deletes a segment of the third chromosome, including the $w r d$ gene (Fig. 4). In $w_{r d}{ }^{104}$ homozygotes, bouton number is decreased by $37 \%$, whereas bouton size is increased $49 \%$ relative to wild type. In $\mathrm{wrd}^{104} / \mathrm{Df}(3 \mathrm{R}) \mathrm{DG} 4$ transheterozygotes, there is an $36 \%$ decrease in bouton number and an $56 \%$ increase in bouton size. The results are not significantly different for the two wrd mutants $(p>0.99$ for both bouton number and size), demonstrating that the phenotype is caused by the loss of $w r d$ and not a second site mutation on the rrd $^{104}$ chromosome. Because fewer synaptic boutons form in wrd mutants, wrd is required for normal synaptic growth at the Drosophila NMJ. The abnormally large bouton size of $w r d$ mutants demonstrates that wrd is also required for normal bouton morphology. In addition to being larger, boutons in $w r d$ mutants often display a more smoothly rounded contour than wild-type boutons (Fig. $4 d, e$ ), leading us to dub this mutation well-rounded (wrd). The well-rounded phenotype of larger boutons and decreased synaptic growth is the opposite of the overexpression phenotype. $w r d$, therefore, is not only required to promote synaptic expansion, but changes in its levels can bidirectionally regulate synaptic morphology and growth.

To understand how wrd regulates synaptic growth, we first need to determine where $w r d$ functions. The protein is present presynaptically at the NMJ but is also present in the postsynaptic muscle. To determine which cell requires $w r d$ expression for normal synaptic growth and morphology, we used the Gal4/UAS system (Brand and Perrimon, 1993) to drive expression of $w r d$ in either nerve or muscle in the $w r d$ mutant background. Neuronal expression of $w$ rd from the elav Gal4 driver rescues the morphological phenotype, leading to a 54\% decrease in bouton size and a $161 \%$ increase in bouton number relative to the $\mathrm{wrd}^{104}$ homozygotes (Fig. $4 b, c$ ). The neuronal rescue of $w r d$ leads to increased bouton number and decreased bouton size relative to wild type, consistent with the previously demonstrated gain-of-function phenotype (Fig. 1b). Such exaggerated rescue suggests that we have not achieved normal levels of Wrd expression, but it does underscore that the level of presynaptic wrd can bidirectionally regulate bouton size and number.

In contrast to neuronal rescue, muscle expression of $w r d$ with the G7 Gal4 driver fails to rescue the loss-of-function bouton number phenotype in the $w r d$ mutant (WT, $19.5 \pm 1.4, n=15$; $w r d^{104} \mathrm{hz}, 11.3 \pm 0.9, n=15$; muscle overexpression, $12.4 \pm 0.9$, 

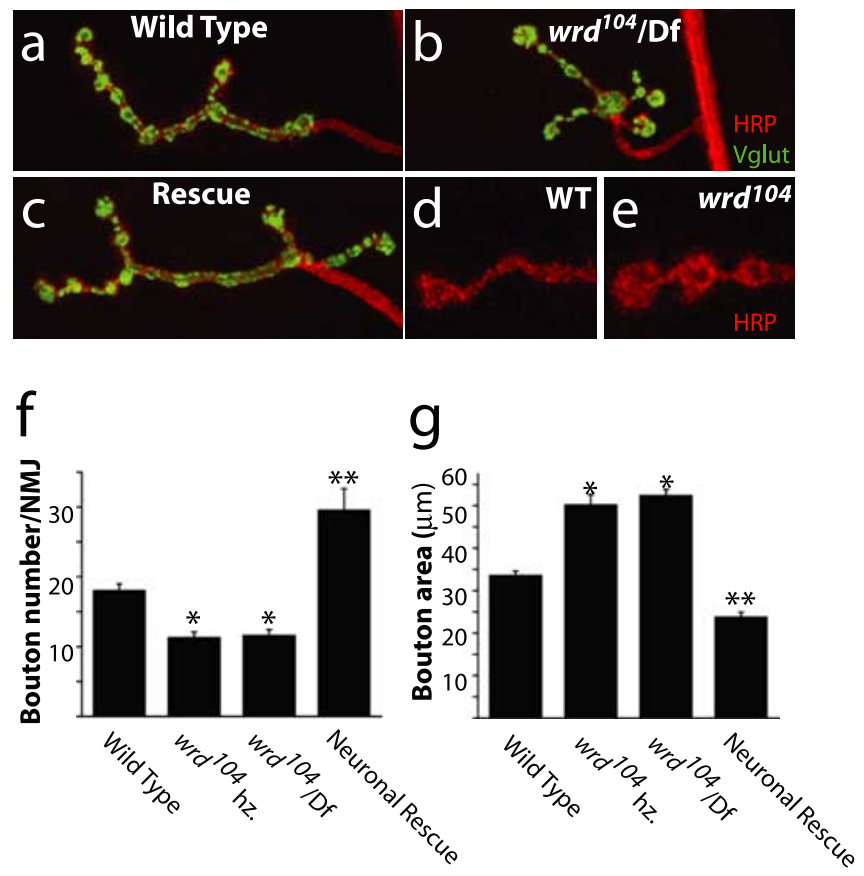

Figure 4. Wrd is required for normal synaptic growth and morphology. $\boldsymbol{a}-\boldsymbol{c}$, Representative NMJs from muscle 4 in wild-type animals $(\boldsymbol{a})$, wrd ${ }^{104} / D f(3 R) D G 4$ mutants $(\boldsymbol{b})$, and wrd $^{104}$ homozygotes rescued with neuronal expression of UAS wrd under elav Gal4 (c). Synapses are costained with antibodies to DVGLUT (green) and HRP (red). $\boldsymbol{d}, \boldsymbol{e}$, Enlarged images of boutons in wild-type animals ( $\boldsymbol{d})$ and wrd $^{104}$ homozygotes $(\boldsymbol{e})$ highlighting increased size and rounded contours of boutons in wrd mutant. $\boldsymbol{f}, \boldsymbol{g}$, Quantification of bouton number and bouton area in segments $A 2$ and $A 3$ of muscle $41 \mathrm{~b}$ innervation of third-instar larvae. Genotypes include wild type (elav Gal4/+), wrd ${ }^{104} / D f(3 R) D G 4$ [elav Gal4/+; wrd $\left.^{104} / \mathrm{Df}(3 \mathrm{R}) D G 4\right), w_{r d} d^{104}$ hz (elav Gal4/+; $w_{r d} d^{104} / w r d^{104}$ )], and neuronally rescued $w r d^{104}$ hz (elav Gal4/UAS wrd; $w_{r d} d^{104} /$ $\left.w r d^{104}\right)$. Bouton number and bouton area in wrd mutants are significantly different from wildtype animals; ${ }^{*} p<0.01$ for bouton number in $4 \mathrm{~b} ;{ }^{*} p<0.001$ for bouton area in $4 c$. wrd $^{104}$ / $D f(3 R) D G 4$ and $w r d^{104} \mathrm{hz}$ are not significantly different for either bouton number or bouton area $(p>0.5)$. Neuronal expression of wrd CDNA rescues both bouton number and bouton area defects in wrd mutants. ${ }^{* *} p<0.01$ for bouton number in $4 \mathrm{~b} ;{ }^{* *} p<0.001$ for bouton area in $4 c$. For both quantifications, wild type, $n=36 ; w^{104} / D f(3 R) D G 4, n=32 ; w^{104}{ }^{104} \mathrm{hz}, n=36$; and rescue, $n=16$. Error bars represent SEM.

$n=16 ; p>0.7)$. Muscle expression does result in a modest reduction in bouton size of the $w r d$ mutant $\left(w r d^{104} \mathrm{hz}, 60.6 \pm 3.1\right.$ $\mu \mathrm{m}$; muscle rescue, $51.8 \pm 2.8 \mu \mathrm{m} ; p<0.05)$, although boutons remain 37\% larger than in wild-type larvae (WT, $37.9 \pm 1.4 \mu \mathrm{m}$; $p<0.001)$. Hence, the primary role of $w r d$ in regulating synapse morphology is in the neuron.

\section{A postsynaptic requirement for $w r d$ in regulating neurotransmitter release}

To assess the physiological consequences of loss of $w r d$ function, we analyzed evoked and spontaneous neurotransmitter release at the NMJ of wild-type, $w r d^{104} / w r d^{104}$, and $w r d^{104} / D f(3 R) D G 4$ third-instar larvae. Both wrd mutants show a large defect in evoked transmitter release (Fig. $5 a, c$ ). This phenotype is not attributable to postsynaptic dysfunction, because there is no significant change in quantal size, the postsynaptic response to the fusion of a single synaptic vesicle (Fig. $5 a, b)[p>0.9$; WT, $n=8$; $\left.w r d^{104} \mathrm{hz}, n=9 ; w r d^{104} / D f(3 R) D G 4, n=8\right]$, and glutamate receptor clusters are aligned opposite active zones (data not shown). Instead, the defect is presynaptic. Estimates of quantal content demonstrate that only half as many synaptic vesicles are released by an action potential at $w r d$ mutant NMJs $(p<0.01)$
(Fig. $5 d$ ). Because wrd functions presynaptically to control synapse morphology and wrd mutants have a presynaptic deficit in transmitter release, we anticipated that neuronal expression of wrd would rescue the physiology phenotype. Instead, we found that neuronal expression of $w r d$ in an otherwise wild-type background leads to a significant decrease in quantal content (Fig. $5 d$ ) $(p<0.01)$, and neuronal expression of $w r d$ in the $w^{104} / w r d^{104}$ mutant fails to rescue $(p>0.8)$. In contrast, muscle expression of wrd rescues the quantal content defect in the $w r d^{104} / w r d^{104} \mathrm{mu}-$ tant (Fig. $5 a, d)$. Hence, postsynaptic wrd is necessary for normal levels of transmitter release from the presynaptic terminal. This implies that $w r d$ participates in an unknown retrograde signaling system from muscle to nerve. When wrd is expressed postsynaptically in the rrd $^{104}$ mutant, it is present throughout the muscle, including at the NMJ (Fig. 5e).

\section{$w r d$ is required for the proper organization of} synaptic microtubules

wrd mutants demonstrate reduced NMJ expansion, a phenotype also observed in several mutants that disrupt microtubules at the Drosophila NMJ, including futsch, fragile X related protein, and aPKC mutants (Roos et al., 2000; Zhang et al., 2001; Ruiz-Canada et al., 2004). Mutants in futsch, a neuron-specific microtubuleassociated protein in Drosophila, are particularly reminiscent of wrd mutants, because futsch mutants also display small NMJs with large boutons (Roos et al., 2000). futsch is a homolog of MAP1b, which stabilizes microtubules and regulates the stability of microtubules in growth cones (Takemura et al., 1992; Goold et al., 1999). The similarity of the futsch and wrd phenotypes suggests that altered microtubular organization could be the molecular mechanism underlying decreased NMJ growth in wellrounded mutants.

To assay microtubule organization, we analyzed Futsch staining, which colocalizes with synaptic $\alpha$-tubulin (Roos et al., 2000). Because Futsch is neuron specific, it provides a clear marker for the microtubule cytoskeleton at the NMJ. Futsch staining marks both bundled and unbundled microtubules at the synapse. Bundled microtubules normally occupy the filamentous core of the nerve terminal. Three types of unbundled microtubules have been described at the NMJ: looped, splayed, or punctate (Packard et al., 2002). In mutants for futsch, decreased microtubule stability is associated with an increased proportion of unbundled microtubule filaments and decreased synaptic expansion (Roos et al., 2000). Thus, whereas unbundled microtubules as assayed by Futsch staining reflect a normal type of microtubule organization, increases in unbundled microtubules correlate with cytoskeletal instability and defects in synaptic growth.

As an index of cytoskeletal structure, we costained larvae for Futsch and DVGLUT, a synaptic marker, and calculated the percentage of Futsch marking unbundled microtubules within the synaptic terminal delineated by DVGLUT. At a wild-type NMJ, Futsch staining highlights bundled microtubules that occupy the core of the synaptic terminal (Fig. $6 a$ ). Most of the fainter Futsch staining is present at terminal boutons, sites containing unbundled microtubules (Roos et al., 2000), although occasional unbundling is observed within synaptic branches (Fig. 6a, arrows). At $w r d$ mutant synapses, however, these instances of unbundling within branches are increased (Fig. $6 a$, arrows). Enlarged images of synaptic branches proximal to the terminal bouton underscore this point, because the wrd mutant branch shows increased unbundled microtubules relative to the wild-type branch (Fig $6 b-$ e). We compared levels of unbundled microtubules in wild-type larvae and $w r d$ mutants. $w r d^{104}$ homozygotes display a $61 \%$ in- 
a
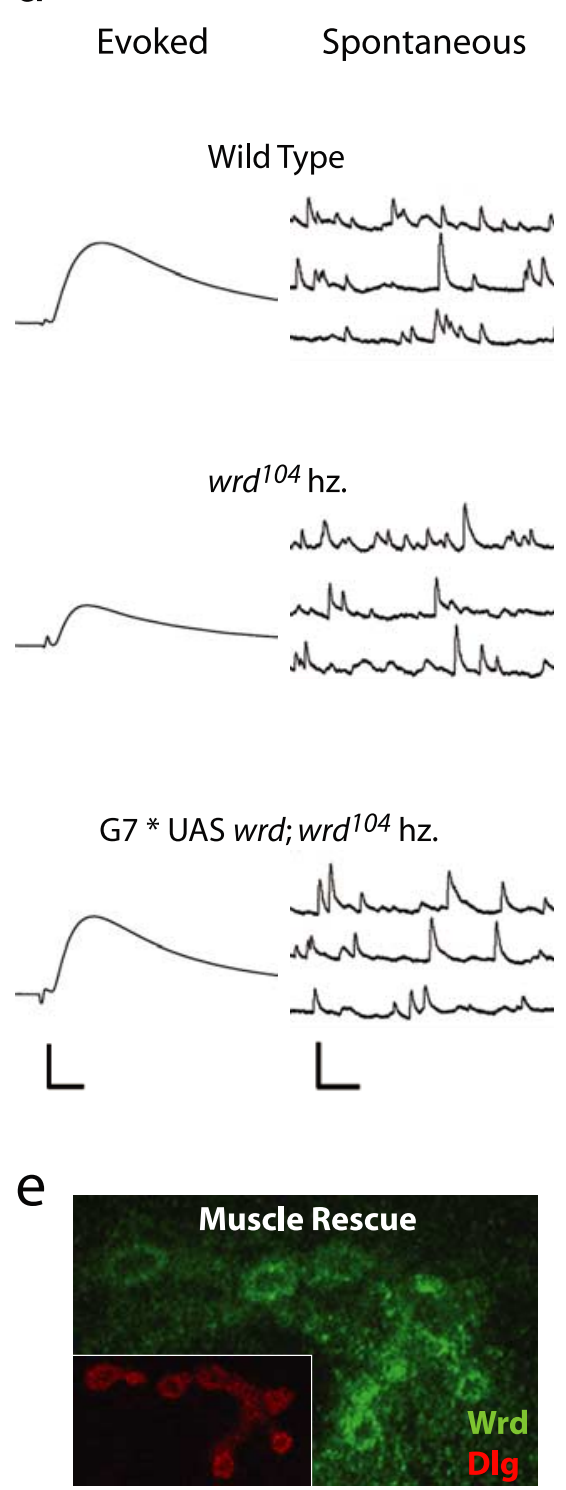

b

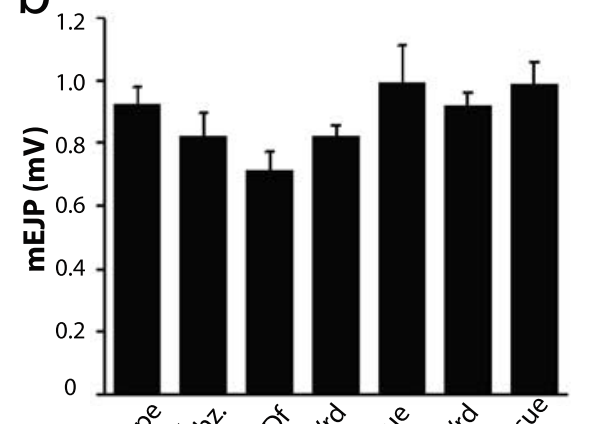

C
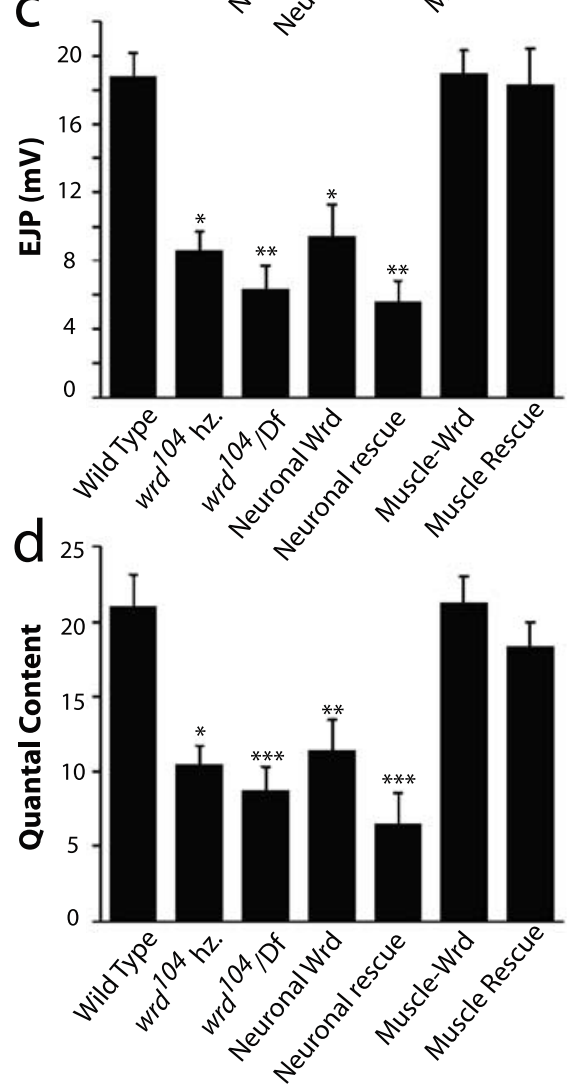

Figure 5. wrd is required postsynaptically for synaptic function. $\boldsymbol{a}$, Representative traces from wild type (elav Gal4/+), $w r d^{104} \mathrm{hz}$ (elav $\mathrm{Gal} / 4$ + ; wrd $^{104} /$ wrd $^{104}$ ), and muscle rescue (G7 Gal4/UAS wrd; wrd $^{104} /$ wrd $^{104}$ ). Calibration: $10 \mathrm{~ms}, 10 \mathrm{mV}$ for evoked release; $250 \mathrm{~ms}, 2$

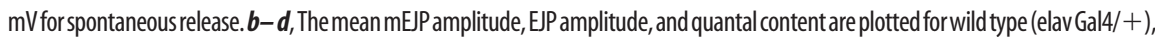
wrd $^{104} \mathrm{hz}$. (elav Gal4/+; wrd $^{104} /$ wrd $^{104}$ ), wrd ${ }^{104} / D f(3 R) D G 4$ (elav Gal4/+; wrd $\left.^{104} / D f(3 R) D G 4\right)$, neuronal Wrd (elav Gal4/UAS wrd), Wrd neuronal rescue (elav Gal4/UAS wrd; wrd ${ }^{104} /$ wrd $^{104}$ ), muscle Wrd (G7 Gal4/UAS wrd), and Wrd muscle rescue (G7 Gal4/UAS wrd; wrd ${ }^{104}$ / $\left.\operatorname{wrd}^{104}\right)\left(n=8\right.$ for wild type and wrd ${ }^{104} / D f(3 R) D G 4 ; n=9$ for wrd ${ }^{104}$ hz and neuronal Wrd; $n=6$ for Wrd neuronal rescue; $n=12$ for muscle Wrd; and $n=10$ for Wrd muscle rescue). mEJPs are not significantly different for any of the genotypes compared with wild type $\left(\right.$ wrd $^{104} / \mathrm{Df}(3 R) D G 4, p>0.3$; all others $\left.p>0.9\right)$. Mean EIP value and quantal content are decreased by 56 and $51 \%$, respectively, in $w_{r d}{ }^{104}$ hz mutants, with similar decreases observed in wrd ${ }^{104} / D f(3 R) D G 4\left[\right.$ wrd $^{104}$ hzand wrd ${ }^{104} / D f(3 R) D G 4$ are not significantly different; $p>0.2$ (EP) and $p>0.3$ (quantal content)]. Muscle expression of Wrd in the wrd ${ }^{104} \mathrm{hz}$ mutants rescues both mean EJP and quantal content to wild-typelevels [wrd ${ }^{104} \mathrm{hz}$ vs muscle rescue, $p<0.05$ (EPP) and $p<0.001$ (quantal content); muscle rescuevs WT, $p>0.8$ (EP) and $p>$ 0.3 (quantal content)]. ${ }^{*} p<0.01$ and ${ }^{* *} p<0.001$ for mean EJP relative to wild type in $c$. In $\boldsymbol{d}^{*} p<0.05,{ }^{* *} p<0.01$, and ${ }^{* * *} p<0.001$ for mean quantal content relative to wild type. Error bars represent SEM. , Wrd staining of NMJ in Wrd muscle rescue (G7 Gal4/UAS wrd; $\mathrm{wrd}^{104} / \mathrm{wrd}^{104}$ ) demonstrates localization of Wrd at the NMJ when expressed in muscle in the absence of endogenous Wrd. Inset shows similar staining pattern with Dlg, a primarily postsynaptic marker.

crease in unbundled microtubules compared with wild-type larvae at the NMJ (Fig. 6h). The $w r d^{104} / D f(3 R) D G 4$ transheterozygotes display a similar increase (Fig. 6h). As a second measure of microtubule organization, we stained for acetylated tubulin, a marker of stabilized microtubules (Zhang et al., 2003). $w r d^{104}$ mutants have a small but significant $12 \%$ decrease in acetylated tubulin intensity (WT intensity, $91.7 \pm$ 3.5; wrd $^{104} /$ wrd $^{104}, 80.8 \pm 3.14 ; n=16$ for both genotypes; $p<0.05$ ) (supplemental Fig. $2 b$, available at www.jneurosci.org as supplemental material). By these two measures, wrd mutants have an abnormal microtubule cytoskeleton at the NMJ. Additionally, neuronal expression of wrd, which rescues the synaptic morphology defect, decreases the proportion of unbundled microtubules in the $w^{104}{ }^{104}$ homozygote $(p<0.05)$ (Fig. 6h). Because wrd regulates microtubule structure, this may explain the role of $w r d$ in promoting synaptic growth. In support of this possibility, we find a genetic interaction between wrd and futsch in the regulation of bouton morphology. Heterozygous mutations for wrd and futsch show no significant increase in bouton size compared with wild type, whereas the transheterozygote has a $31 \%$ increase in bouton size $(p<0.01 ; n=12)$. No transheterozygous interaction was identified for bouton number $(p>0.7)$. This genetic interaction is consistent with the hypothesis that wrd affects synaptic morphology, at least in part, by regulating microtubule organization.

PP2A activity is essential for stability of the NMJ cytoskeleton

The requirement of a PP2A regulatory subunit for microtubule organization at the synapse suggests that PP2A activity regulates the synaptic cytoskeleton. To test whether PP2A activity is required for cytoskeletal structure, we asked whether decreasing the catalytic activity of PP2A affects the organization of synaptic microtubules. We reduced PP2A activity by targeting the single PP2A catalytic subunit in Drosophila, mts. As the sole catalytic subunit, $m t s$ interacts with all PP2A regulatory subunits to mediate the catalysis of PP2A. Homozygous mutants in $m t s$ are embryonic lethal because cells are unable to complete mitosis (Snaith et al., 1996). To circumvent this early lethality, we inhibited PP2A activity using a well characterized dominant-negative transgene of $m t s$ (UAS dnMts) that binds to the structural and regulatory subunits of PP2A but lacks the active site of the enzyme (Hannus et al., 2002; Sathyanarayanan et al., 2004). We expressed the UAS dnMts transgene under the neuronal elav Gal4 driver to restrict the inhibition of PP2A activity to postmitotic neurons, thereby avoiding lethality from disruptions in mitosis. Neuronal expression of the dominant-negative $m t s$ results in a dramatic disruption of the cytoskeleton at the NMJ (Fig. 7a), 
a
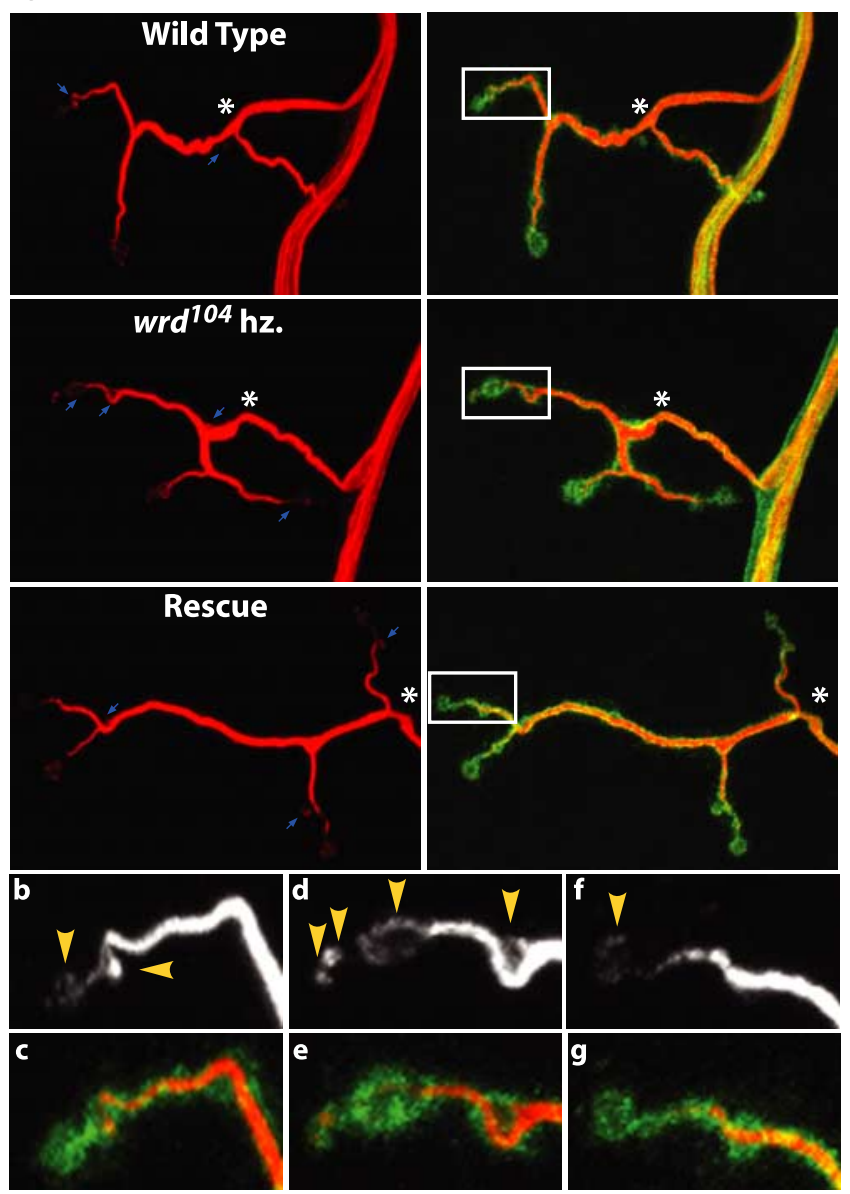

$\mathrm{h}$

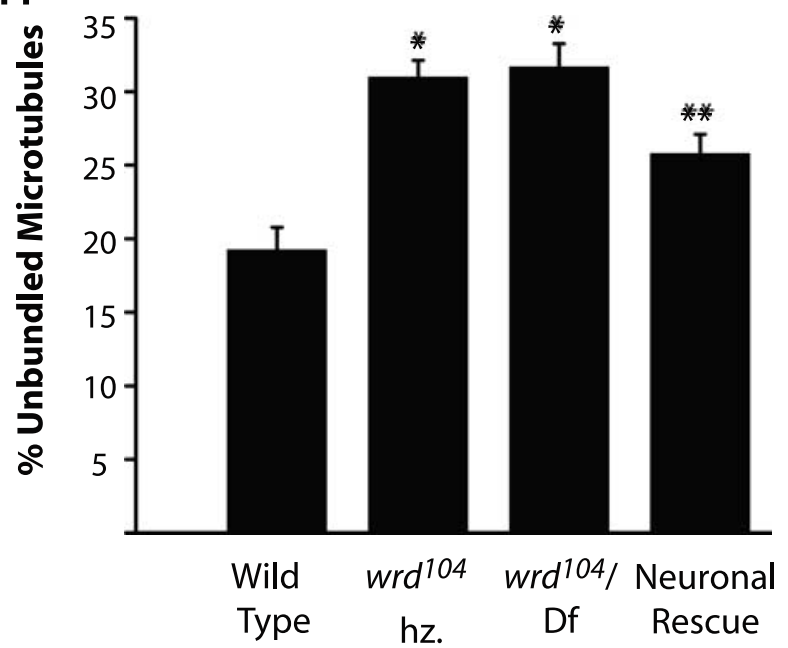

Figure 6. Loss of wrd disrupts cytoskeletal stability. $\boldsymbol{a}$, Representative confocal images of muscle 4 synapses from wild type (elav Gal4/+), wrd ${ }^{104} \mathrm{hz}$ (elav Gal4/+; wrd $^{104} /$ wrd $^{104}$ ), and rescue (elav Gal4/UAS wrd; wrd ${ }^{104} / w^{\prime} d^{104}$ ). Left column displays synapses stained with antibody to Futsch, a reporter for microtubules, and right column displays corresponding synapses costained with $\alpha$-Futsch and $\alpha$-HRP. Futsch staining demonstrates bundled microtubules within the core of the synaptic terminal. Unbundled microtubules are visible at terminal boutons and, in some instances, along the shaft of the terminal, as marked by arrows. The wrd mutant shows an increased occurrence of unbundled microtubules along the shaft of the terminal. Asterisks mark junction between the synaptic terminal and preterminal axon. Insets mark a representative sample of unbundled microtubules at terminal boutons and are enlarged in $\boldsymbol{b}-\boldsymbol{g} . \boldsymbol{b}-\boldsymbol{g}$, Enlarged insets displaying representative terminal boutons from wild type $(\boldsymbol{b}, \boldsymbol{c})$, with an $88 \%$ increase in the levels of unbundled microtubules, as assayed by Futsch staining, relative to wild type after inhibition of PP2A catalysis. Boutons are observed that are filled with an unusually high number of splayed filaments (Fig. $7 a$, arrows), and the core region of several branches display attenuated Futsch staining. This disorganized cytoskeleton is accompanied by an aberrant NMJ morphology, with the presence of occasional extremely large boutons as well as the thin tendrils of very small boutons (Fig. 7a). Overexpression of the wild-type $m t s$ transgene, which has been shown to promote PP2A activity (Sathyanarayanan et al., 2004), has no effect on the proportion of unbundled microtubules $(p>0.6)$. PP2A activity is therefore required but not limiting for normal cytoskeletal organization at the NMJ.

Because inhibiting both the $m t s$ catalytic subunit and the wrd regulatory subunit disrupts the synaptic cytoskeleton, Wrd likely functions to promote PP2A activity. This hypothesis predicts that loss-of-function wrd mutants should enhance the mts phenotype. To test for a genetic interaction between wrd and $m t s$, we used $w r d^{104}$ heterozygous mutants (het). We found no interaction with an $m t s$ heterozygous loss-of-function mutant but did with neuronal expression of the dominant-negative $m t s$ transgene. When only one of two genomic copies of wrd is disrupted, there is no significant effect on microtubule unbundling in an otherwise wild-type background ( $p>0.6$ ). However, the $\mathrm{wrd}^{104}$ heterozygote significantly enhances the unbundling phenotype of neuronal dominant-negative $m t s$ expression (Fig. $7 b$ ), leading to a $46 \%$ increase in unbundled microtubules relative to inhibition of $m t s$ alone $(p<0.01)$. In addition, the cytoskeletal morphology of these double mutants is more severely disrupted than under the dnMts condition. Whereas the filamentous microtubule core is attenuated when $m t s$ is inhibited, simultaneously inhibiting $m t s$ and wrd results in synapses in which microtubules are splayed or punctate throughout the normally stable core region of the synaptic terminal (Fig. 7a). This genetic interaction demonstrates that wrd promotes PP2A function and suggests that wrd positively regulates the PP2A holoenzyme to stabilize the synaptic cytoskeleton.

\section{Discussion}

Regulation of synaptic growth and morphology are essential for the development and refinement of neural circuits. Here we show that well-rounded, a regulatory subunit of protein phosphatase $2 \mathrm{~A}$, is required for normal growth and morphology of the Drosophila neuromuscular junction. We identified wrd in a gain-offunction screen for candidate mediators of synaptic growth. Overexpression of Wrd protein in neurons leads to overgrowth of the NMJ and a decrease in the size of synaptic boutons. Although the Wrd protein is widely expressed, it is enriched in the synaptic neuropil and at the NMJ. We generated null mutants for $w r d$ and find that presynaptic expression of wrd is required for normal

$w_{r d}{ }^{104} \mathrm{hz}(\boldsymbol{d}, \boldsymbol{e})$, and rescue $(\boldsymbol{f}, \boldsymbol{g})$. Top row shows staining for Futsch alone, and bottom row shows costaining of Futsch and HRP. Arrowheads in top row indicate instances of unbundled microtubules. Although insets display a similar number of boutons for each genotype, instances of unbundled microtubules are greatest in the $\mathrm{wrd}^{104}$ mutant and take up a greater proportion of the total microtubule area than in the other genotypes. c, Quantification of unbundled microtubules as a percentage of total area of synaptic microtubules. For wild type (elav Gal4/+), wrd $^{104}$ hz (elav Gal4/+; wrd $^{104} /$ wrd $^{104}$ ), wrd ${ }^{104} / D f(3 R) D G 4$ [elav Gal4/+; wrd $^{104} /$ Df(3R)DG4], and neuronal rescue (elav Gal4/UAS wrd; wrd ${ }^{104} /$ wrd $^{104}$ ), $n=27,27,23$, and 28, respectively. Both wrd mutants have increased unbundled microtubules relative to $W T ;{ }^{*} p<0.01$. Neuronal rescue significantly decreases unbundled microtubules relative to both wrd mutants; ${ }^{* *} p<$ 0.05. Error bars indicate SEM. 
a
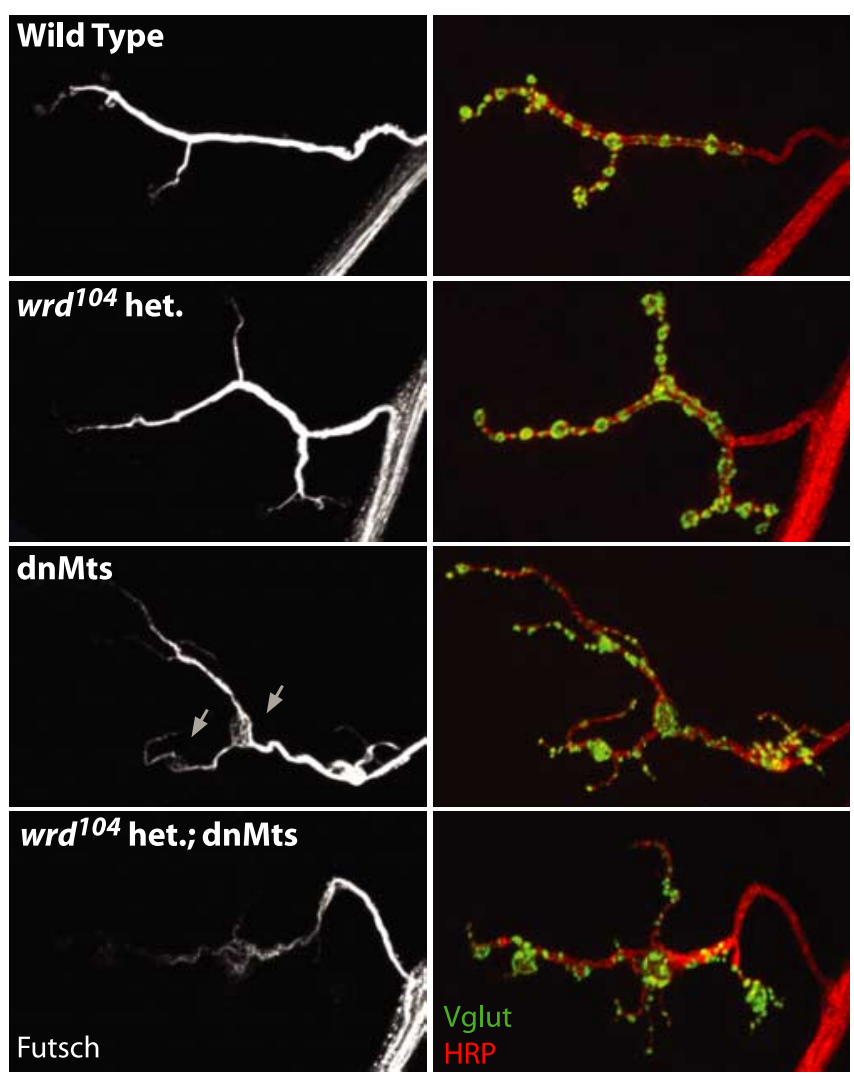

b

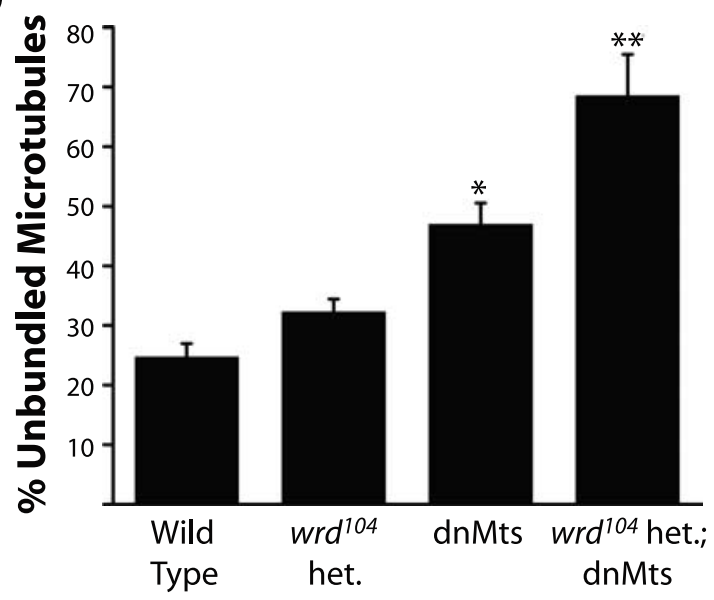

Figure 7. PP2A activity is required for cytoskeletal stability of synapse. $\boldsymbol{a}$, Representative confocal images of muscle 4 synapses from wild type (elav Gal4/+), wrd ${ }^{104}$ het (elav Gal4/+ ; $w_{r d}{ }^{104} /+$ ), dnMts (elav Gal4/UAS dnMts), and dnMts; wrd $^{104}$ het (elav Gal4/+; wrd $^{104} /$ UAS $\mathrm{dnMts}$ ). Left column shows synapses labeled with Futsch, and right column shows corresponding synapses labeled with $\alpha$-DVGLUT and $\alpha$-HRP. In the elav dnMts case, arrows mark boutons with dramatic unbundling of microtubules. $\boldsymbol{b}$, Quantification of percentage unbundled microtubules at NMJ. Unbundled microtubules are measured as a proportion of total area of microtubules at synaptic terminal. $n=19,14,27,16$ for WT, wrd ${ }^{104}$ het, dnMts, and wrd ${ }^{104}$ het; dnMts, respectively. Reduced PP2A activity leads to significantly increased microtubule unbundling relative to wild type; ${ }^{*} p<0.05$. The combined wrd $^{104}$ het; dnMts mutant shows a significant increase in unbundled microtubules relative to dnMts alone; ${ }^{* *} p<0.01$. The heterozygous wrd mutant thus enhances the effect of reduced PP2A activity, suggesting that it can positively regulate the activity of PP2A toward the synaptic cytoskeleton. Error bars indicate SEM. synaptic growth and morphology. Postsynaptic expression of $w r d$ is required for normal levels of evoked neurotransmitter release. An increased proportion of unbundled synaptic microtubules accompanies the defect in NMJ morphology, suggesting a potential molecular mechanism for the aberrant synapse morphology. High levels of unbundled microtubules are also observed when the catalytic activity of PP2A is inhibited, an effect enhanced by reducing the expression of $w r d$. Hence, both the regulatory wrd subunit and the catalytic subunit of PP2A are required for normal cytoskeletal organization at the Drosophila NMJ. Our findings demonstrate that well-rounded is required for normal synaptic growth and morphology at the Drosophila NMJ and suggest that well-rounded regulates the cytoskeleton by promoting PP2A activity.

\section{Regulation of synaptic morphology and growth by PP2A and well-rounded}

Protein phosphatase $2 \mathrm{~A}$ is a ubiquitous serine-threonine phosphatase required for viability in mammals and Drosophila. PP2A comprises three subunits: a core enzyme consisting of a structural and catalytic subunit and a variable regulatory subunit, which can regulate activity, substrate specificity, and subcellular localization of the core enzyme (Janssens and Goris, 2001). Although the core enzyme is expressed in all tissues, it is highly enriched in both the vertebrate and Drosophila nervous system (Mayer et al., 1991; Mayer-Jaekel and Hemmings, 1995; Strack et al., 1998; Zolnierowicz et al., 2004). In mice, $\mathrm{B}^{\prime}$ regulatory subunits are also highly enriched in the brain, especially during development (Martens et al., 2004). We observe similar results with the Drosophila B' subunit well-rounded. Wrd protein is present in many tissues but is enriched in the larval nervous system, especially in the synaptic neuropil and the NMJ.

Although PP2A is highly expressed in the developing nervous system, relatively little is known about its role in nervous system development, particularly at the cellular level. In vivo models have demonstrated a role for PP2A in differentiation of neural structures, such as forebrain and midbrain in Xenopus (Yang et al., 2003) and sensory organs in Drosophila (Shiomi et al., 1994). Evidence for a role in controlling synaptic structure comes from pharmacological studies in cultured cells, in which treatment with okadaic acid, an inhibitor of both PP2A and protein phosphatase 1 (PP1), leads to a decrease in neuronal processes and number of synapses (Tapia et al., 1999). Our work at the Drosophila $\mathrm{NMJ}$ demonstrates in vivo that $\mathrm{PP} 2 \mathrm{~A}$ and a specific $\mathrm{PP} 2 \mathrm{~A}$ regulatory subunit are required for the normal morphological development of synapses.

\section{Wrd and PP2A catalytic activity regulate the synaptic cytoskeleton}

Loss of $w r d$ and inhibition of PP2A catalytic activity both increase the proportion of unbundled synaptic microtubules as assayed by staining for Futsch, a homolog of the microtubule-associated protein MAP1B, which promotes microtubule stability (Takemura et al., 1992; Goold et al., 1999). Similar Futsch localization is associated with a destabilized microtubule cytoskeleton and defects in synaptic growth (Roos et al., 2000; Zhang et al., 2001; da Cruz et al., 2005). Phosphorylation dynamics are known to play an important role in cytoskeletal stability. For example, phosphorylation of MAP1B inhibits its stabilizing function, because phosphorylated MAP1B leads to a reduction of stable microtubules in growth cones (Goold et al., 1999). Work in cultured neurons demonstrates that inhibition of PP2A and PP1 with okadaic acid disrupts microtubules, demonstrating a requirement 
for dephosphorylation by PP2A and/or PP1 for stability of the neuronal cytoskeleton (Merrick et al., 1997; Tapia et al., 1999). PP2A also regulates the dephosphorylation of MAP1B (Gong et al., 2000), a potential mechanism for the cytoskeletal disruption observed with inhibition of PP2A activity. Our findings support and extend these observations by demonstrating a requirement for PP2A catalysis and a B' regulatory subunit for normal synaptic cytoskeletal organization in vivo. The reduced stabilizing function of phosphorylated MAP1B, the similarity of futsch and wrd mutant phenotypes, and the genetic interaction between futsch and $w r d$ are consistent with the model that Futsch or other MAPs are substrates for the PP2A at the Drosophila NMJ. In this model, reduced PP2A function would lead to hyperphosphorylation of MAPs and microtubule destabilization, culminating in abnormal synaptic morphology and impaired synaptic growth.

\section{Wrd and other PP2A regulatory subunits in Drosophila}

In addition to $w r d$, four other PP2A regulatory subunit genes have been identified in Drosophila. Two of these have been characterized: twins, a B regulatory subunit, and $w d b$, the second $\mathrm{B}^{\prime}$ subunit in Drosophila. Nulls for twins and $w d b$ are lethal (Uemura et al., 1993; Hannus et al., 2002), making well-rounded the first nonessential subunit characterized in Drosophila. twins is expressed in the nervous system (Mayer-Jaekel et al., 1993), in which it is required for normal differentiation of sensory organ structures (Shiomi et al., 1994). The expression of $w d b$ in the nervous system has not been characterized, although neuronal overexpression of $w d b$ disrupts sensory organ formation and axon guidance (Abdelilah-Seyfried et al., 2000; Kraut et al., 2001). Because mutants of each regulatory subunit have distinct loss-offunction phenotypes, these subunits are not redundant. However, inhibiting the catalytic subunit disrupts the synaptic cytoskeleton more severely than does a null mutant for the regulatory $w r d$ subunit, implying that other regulatory subunits must also mediate some PP2A function at the synapse. As a second $\mathrm{B}^{\prime}$ subunit, widerborst is a logical candidate to overlap in function with $w r d$. However, unlike $w r d$, we find that neuronal overexpression of widerborst does not lead to synaptic overgrowth [mean bouton number per NMJ: WT (elav/+), $16.7 \pm$ 16; elav/UAS Wdb, $17.5 \pm 16 ; p>0.6$ ]. Furthermore, we find no enhancement of the wrd phenotype by expressing a dominantnegative widerborst transgene (Hannus et al., 2002) in motoneurons (mean bouton number per NMJ: $w r d^{104} \mathrm{hz}, 14.6 \pm 14$; elav/ UAS dnWdb, $w_{r d}{ }^{104} \mathrm{hz}, 14.3 \pm 16 ; p>0.8$; mean bouton area: $w{ }^{104} \mathrm{hz}, 48.0 \pm 2.3 \mu \mathrm{m}, n=14$; elav/UAS dnWdb, $w_{r d}{ }^{104} \mathrm{hz}$, $46.4 \pm 2.1 \mu \mathrm{m}, n=16 ; p>0.5)$. Hence, our characterization of wrd demonstrates a specific role for a particular $\mathrm{B}^{\prime}$ subunit in synaptic development, although the residual PP2A activity at $w r d$ mutant synapses must require other regulatory subunits.

\section{Postsynaptic $w r d$ regulates presynaptic neurotransmitter release}

Synaptic strength is decreased in wrd mutants attributable to a decrease in quantal content, the number of vesicles released by the nerve. Quantal content is a presynaptic property, reflecting the number of presynaptic release sites and the probability of release from each site. Although presynaptic wrd expression rescues the $w r d$ synapse morphology phenotypes, postsynaptic $w r d$ expression rescues the defect in quantal content. This suggests that $w r d$ is required for a retrograde signal controlling presynaptic release. Activity-dependent retrograde signals that respond to changes in quantal size have been described previously at the Drosophila NMJ (Petersen et al., 1997; DiAntonio et al., 1999;
Paradis et al., 2001). In wrd mutants, however, quantal size is normal. Hence, if $w r d$ functions in this same retrograde signaling pathway, it must function downstream of glutamate receptor activity and membrane depolarization. In this model, PP2Adependent dephosphorylation of a component of the homeostatic retrograde signaling pathway would be required for appropriate regulation of presynaptic release characteristics.

\section{Regulation of synaptic stability in development and disease} PP2A activity and the phosphorylation state of MAPs have been implicated in the pathogenesis of neurodegenerative disease. Hyperphosphorylated tau, an MAP, accumulates in intracellular neurofibrillary tangles and is associated with neurodegeneration and neuronal death in Alzheimer's disease. PP2A dephosphorylates MAPs, including tau (Ulloa et al., 1993; Saito et al., 1995; Gong et al., 2000), and reduced amounts of PP2A mRNA and protein are present in postmortem brains of Alzheimer's patients (Vogelsberg-Ragaglia et al., 2001; Sontag et al., 2004). Our data now demonstrate a physiological requirement for PP2A in organizing the synaptic cytoskeleton. In the most severe mutant, in which the PP2A catalytic and the wrd regulatory subunits are simultaneously inhibited, we observe a highly attenuated cytoskeleton with barely visible MAP staining. Thus, interfering with PP2A during development provokes features reminiscent of the synaptic and axonal loss that may precede cell death in neurodegenerative disease (Coleman and Yao, 2003; Scheff and Price, 2003). Additional dissection of PP2A function at the synapse could enhance our understanding of mechanisms controlling synaptic structure during development and disease.

\section{References}

Abdelilah-Seyfried S, Chan Y-M, Zeng C, Justice NJ, Younger-Shepherd S, Sharp LE, Barbel S, Meadows SA, Jan LY, Jan YN (2000) A gain-offunction screen for genes that affect the development of the Drosophila adult external sensory organ. Genetics 155:733-752.

Barnes GN, Slevin JT, Vanaman TC (1995) Rat brain protein phosphatase 2A: an enzyme that may regulate autophosphorylated protein kinases. J Neurochem 64:340-353.

Belmeguenai A, Hansel C (2005) A role for protein phosphatases 1, 2A, and 2B in cerebellar long-term potentiation. J Neurosci 25:10768-10772.

Betz WJ, Henkel AW (1994) Okadaic acid disrupts clusters of synaptic vesicles in frog motor nerve terminals. J Cell Biol 124:843-854.

Brand AH, Perrimon N (1993) Targeted gene expression as a means of altering cell fates and generating dominant phenotypes. Development 118:401-415.

Coleman PD, Yao PJ (2003) Synaptic slaughter in Alzheimer's disease. Neurobiol Aging 24:1023-1027.

da Cruz AB, Schwarzel M, Schulze S, Niyyati M, Heisenberg M, Kretzschmar D (2005) Disruption of the MAP1B-related protein FUTSCH leads to changes in the neuronal cytoskeleton, axonal transport defects, and progressive neurodegeneration in Drosophila. Mol Biol Cell 16:2433-2442.

Daniels RW, Collins CA, Gelfand MV, Dant J, Brooks ES, Krantz DE, DiAntonio A (2004) Increased expression of the Drosophila vesicular glutamate transporter leads to excess glutamate release and a compensatory decrease in quantal content. J Neurosci 24:10466-10474.

DiAntonio A, Petersen SA, Heckmann M, Goodman CS (1999) Glutamate receptor expression regulates quantal size and quantal content at the Drosophila neuromuscular junction. J Neurosci 19:3023-3032.

DiAntonio A, Haghighi AP, Portman SL, Lee JD, Amaranto AM, Goodman CS (2001) Ubiquitination-dependent mechanisms regulate synaptic growth and function. Nature 412:449-452.

Gong C-X, Wegiel J, Lidsky T, Zuck L, Avila J, Wisniewski HM, GrundkeIqbal I, Iqbal K (2000) Regulation of phosphorylation of neuronal microtubule-associated proteins MAP1b and MAP2 by protein phosphatase-2A and -2B in rat brain. Brain Res 853:299-309.

Goold RG, Owen R, Gordon-Weeks PR (1999) Glycogen synthase kinase 3beta phosphorylation of microtubule-associated protein $1 \mathrm{~B}$ regulates the stability of microtubules in growth cones. J Cell Sci 112:3373-3384. 
Hannus M, Feiguin F, Heisenberg C-P, Eaton S (2002) Planar cell polarization requires Widerborst, a $\mathrm{B}^{\prime}$ regulatory subunit of protein phosphatase 2A. Development 129:3493-3503.

Hoang B, Chiba A (2001) Single-cell analysis of Drosophila larval neuromuscular synapses. Dev Biol 229:55-70.

Janssens V, Goris J (2001) Protein phosphatase 2A: a highly regulated family of serine/threonine phosphatases implicated in cell growth and signalling. Biochemical J 353:417-439.

Jouvenceau A, Billard J-M, Haditsch U, Mansuy IM, Dutar P (2003) Different phosphatase-dependent mechanisms mediate long-term depression and depotentiation of long-term potentiation in mouse hippocampal CA1 area. Eur J Neurosci 18:1279-1285.

Kraut R, Menon K, Zinn K (2001) A gain-of-function screen for genes controlling motor axon guidance and synaptogenesis in Drosophila. Curr Biol 11:417-430.

Marrus SB, DiAntonio A (2004) Preferential localization of glutamate receptors opposite sites of high presynaptic release. Curr Biol 14:924-931.

Marrus SB, Portman SL, Allen MJ, Moffat KG, DiAntonio A (2004) Differential localization of glutamate receptor subunits at the Drosophila neuromuscular junction. J Neurosci 24:1406-1415.

Martens E, Stevens I, Janssens V, Vermeesch J, Gotz J, Goris J, Van Hoof C (2004) Genomic organisation, chromosomal localisation tissue distribution and developmental regulation of the PR61/B'; regulatory subunits of protein phosphatase 2A in mice. J Mol Biol 336:971-986.

Mayer R, Hendrix P, Cron P, Matthies R, Stone S, Goris J, Merlevede W, Hofsteenge J, Hemmings BA (1991) Structure of the 55-kDa regulatory subunit of protein phosphatase 2A: evidence for a neuronal-specific isoform. Biochemistry 30:3589-3597.

Mayer-Jaekel RE, Hemmings BA (1995) Role of protein phosphatase 2A in Drosophila development. Semin Cancer Biol 6:249-256.

Mayer-Jaekel RE, Ohkura H, Gomes R, Sunkel CE, Baumgartner S, Hemmings BA, Glover DM (1993) The $55 \mathrm{kd}$ regulatory subunit of Drosophila protein phosphatase 2A is required for anaphase. Cell 72:621-633.

Merrick SE, Trojanowski JQ, Lee VMY (1997) Selective destruction of stable microtubules and axons by inhibitors of protein serine/threonine phosphatases in cultured human neurons (NT2N cells). J Neurosci 17:5726-5737.

Packard M, Koo ES, Gorczyca M, Sharpe J, Cumberledge S, Budnik V (2002) The Drosophila Wnt, Wingless, provides an essential signal for pre- and postsynaptic differentiation. Cell 111:319-330.

Paradis S, Sweeney ST, Davis GW (2001) Homeostatic control of presynaptic release is triggered by postsynaptic membrane depolarization. Neuron 30:737-749.

Petersen SA, Fetter RD, Noordermeer JN, Goodman CS, DiAntonio A (1997) Genetic analysis of glutamate receptors in Drosophila reveals a retrograde signal regulating presynaptic transmitter release. Neuron 19:1237-1248.

Price NE, Wadzinski B, Mumby MC (1999) An anchoring factor targets protein phosphatase 2A to brain microtubules. Mol Brain Res 73:68-77.

Roos J, Hummel T, Ng N, Klambt C, Davis GW (2000) Drosophila Futsch regulates synaptic microtubule organization and is necessary for synaptic growth. Neuron 26:371-382.

Rorth P, Szabo K, Bailey A, Laverty T, Rehm J, Rubin GM, Weigmann K, Milan M, Benes V, Ansorge W, Cohen SM (1998) Systematic gain-offunction genetics in Drosophila. Development 125:1049-1057.

Ruiz-Canada C, Ashley J, Moeckel-Cole S, Drier E, Yin J, Budnik V (2004) New synaptic bouton formation is disrupted by misregulation of microtubule stability in aPKC mutants. Neuron 42:567-580.

Saito T, Ishiguro K, Uchida T, Miyamoto E, Kishimoto T, Hisanaga S-I (1995) In situ dephosphorylation of tau by protein phosphatase $2 \mathrm{~A}$ and 2B in fetal rat primary cultured neurons. FEBS Lett 376:238-242.

Sathyanarayanan S, Zheng X, Xiao R, Sehgal A (2004) Posttranslational regulation of Drosophila PERIOD protein by protein phosphatase 2A. Cell 116:603-615.

Scheff SW, Price DA (2003) Synaptic pathology in Alzheimer's disease: a review of ultrastructural studies. Neurobiol Aging 24:1029-1046.

Shiomi K, Takeichi M, Nishida Y, Nishi Y, Uemura T (1994) Alternative cell fate choice induced by low-level expression of a regulator of protein phosphatase 2A in the Drosophila peripheral nervous system. Development 120:1591-1599.

Snaith HA, Armstrong CG, Guo Y, Kaiser K, Cohen PT (1996) Deficiency of protein phosphatase $2 \mathrm{~A}$ uncouples the nuclear and centrosome cycles and prevents attachment of microtubules to the kinetochore in Drosophila microtubule star (mts) embryos. J Cell Sci 109:3001-3012.

Sontag E, Nunbhakdi-Craig V, Bloom GS, Mumby MC (1995) A novel pool of protein phosphatase $2 \mathrm{~A}$ is associated with microtubules and is regulated during the cell cycle. J Cell Biol 128:1131-1144.

Sontag E, Nunbhakdi-Craig V, Lee G, Bloom GS, Mumby MC (1996) Regulation of the phosphorylation state and microtubule-binding activity of tau by protein phosphatase 2A. Neuron 17:1201-1207.

Sontag E, Nunbhakdi-Craig V, Lee G, Brandt R, Kamibayashi C, Kuret J, White CL, III, Mumby MC, Bloom GS (1999) Molecular interactions among protein phosphatase $2 \mathrm{~A}$, tau, and microtubules. Implications for the regulation of tau phosphorylation and the development of tauopathies. J Biol Chem 274:25490-25498.

Sontag E, Luangprom A, Hladik C, Mudrak I, Ogris E, Speciale S, White CL, III (2004) Altered expression levels of the protein phosphatase $2 \mathrm{~A} \mathrm{AB} \alpha \mathrm{C}$ enzyme are associated with Alzheimer disease pathology. J Neuropathol Exp Neurol 63:287-301.

Stewart BA, Atwood HL, Renger JJ, Wang J, Wu CF (1994) Improved stability of Drosophila larval neuromuscular preparations in haemolymphlike physiological solutions. J Comp Physiol A Neuroethol Sens Neural Behav Physiol 175:179-191.

Strack S, Zaucha JA, Ebner FF, Colbran RJ, Wadzinski BE (1998) Brain protein phosphatase $2 \mathrm{~A}$ : developmental regulation and distinct cellular and subcellular localization by B subunits. J Comp Neurol 392:515-527.

Takemura R, Okabe S, Umeyama T, Kanai Y, Cowan NJ, Hirokawa N (1992) Increased microtubule stability and alpha tubulin acetylation in cells transfected with microtubule-associated proteins MAP1B, MAP2 or tau. J Cell Sci 103:953-964.

Tapia R, Pena F, Arias C (1999) Neurotoxic and synaptic effects of okadaic acid, an inhibitor of protein phosphatases. Neurochem Res 24:1423-1430.

Toba G, Ohsako T, Miyata N, Ohtsuka T, Seong K-H, Aigaki T (1999) The gene search system: a method for efficient detection and rapid molecular identification of genes in Drosophila melanogaster. Genetics 151:725-737.

Uemura T, Shiomi K, Togashi S, Takeichi M (1993) Mutation of twins encoding a regulator of protein phosphatase $2 \mathrm{~A}$ leads to pattern duplication in Drosophila imaginal discs. Genes Dev 7:429-440.

Ulloa L, Dombradi V, Diaz-Nido J, Szucs K, Gergely P, Friedrich P, Avila J (1993) Dephosphorylation of distinct sites on microtubule-associated protein MAP1B by protein phosphatases $1,2 \mathrm{~A}$ and $2 \mathrm{~B}$. FEBS Lett 330:85-89.

Vogelsberg-Ragaglia V, Schuck T, Trojanowski JQ, Lee VMY (2001) PP2A mRNA expression is quantitatively decreased in Alzheimer's disease hippocampus. Exp Neurol 168:402-412.

Wagh DA, Rasse TM, Asan E, Hofbauer A, Schwenkert I, Durrbeck H, Buchner S, Dabauvalle M-C, Schmidt M, Qin G (2006) Bruchpilot, a protein with homology to ELKS/CAST, is required for structural integrity and function of synaptic active zones in Drosophila. Neuron 49:833-844.

Woo NH, Nguyen PV (2002) "Silent" metaplasticity of the late phase of long-term potentiation requires protein phosphatases. Learn Mem 9:202-213.

Wu C, Wairkar YP, Collins CA, DiAntonio A (2005) Highwire function at the Drosophila neuromuscular junction: spatial, structural, and temporal requirements. J Neurosci 25:9557-9566.

Yang J, Wu J, Tan C, Klein PS (2003) PP2A:B56 $\varepsilon$ is required for Wnt $/ \beta$ catenin signaling during embryonic development. Development 130:5569-5578.

Yao KM, White K (1994) Neural specificity of Elav expression: defining a Drosophila promoter for directing expression to the nervous system. J Neurochem 63:41-51.

Zhang Y, Li N, Caron C, Matthias G, Hess D, Khochbin S, Matthias P (2003) HDAC-6 interacts with and deacetylates tubulin and microtubules in vivo. EMBO J 22:1168-1179.

Zhang YQ, Bailey AM, Matthies HJG, Renden RB, Smith MA, Speese SD, Rubin GM, Broadie K (2001) Drosophila fragile X-related gene regulates the MAP1B homolog Futsch to control synaptic structure and function. Cell 107:591-603.

Zolnierowicz S, Csortos C, Bondor J, Verin A, Mumby MC, De-Paoli-Roach A (2004) Diversity in the regulatory B-subunits of protein phosphatase 2A: identification of a novel isoform highly expressed in brain. Biochemistry $3: 287-301$. 